\title{
Partition Coefficients of Organic Compounds in Four New Tetraalkylammonium Bis(trifluoromethylsulfonyl)imide Ionic Liquids Using Inverse Gas Chromatography
}

\author{
William E. Acree, Jr., ${ }^{* \dagger}$ Gary A. Baker, ${ }^{\ddagger}$ Fabrice Mutelet, ${ }^{\S}$ and Jean-Charles Moise ${ }^{\S}$ \\ ${ }^{\dagger}$ Department of Chemistry, 1155 Union Circle \#305070, University of North Texas, Denton, Texas 76203-5017, United States \\ ${ }^{\ddagger}$ Department of Chemistry, University of Missouri - Columbia, Columbia, Missouri 65211, United States \\ §Laboratoire de Réactions et Génie des Procédés, Nancy-Université, 1 rue Grandville, BP 2045154001 Nancy, France
}

\begin{abstract}
The activity coefficients at infinite dilution, $\gamma_{1,2}^{\infty}$, of 47 solutes including various (cyclo)alkanes, alkenes, alkynes, aromatic hydrocarbons, alcohols, water, thiophene, ethers, aldehydes, and ketones, in the four ionic liquids (ILs) decyl(trimethyl)ammonium bis(trifluoromethylsulfonyl)imide, methyl(tributyl)ammonium bis(trifluoromethylsulfonyl)imide, octyl(trimethyl)ammonium bis(trifluoromethylsulfonyl)imide, and tetraoctylammonium bis(trifluoromethylsulfonyl)imide, were measured using inverse gas chromatography at temperatures from (323 to 343) K. The retention data were also converted to gas-to-IL and water-to-IL partition coefficients using the corresponding gas-to-water partition coefficients. Both sets of partition coefficients were analyzed using the modified Abraham solvation parameter model. The derived equations correlated the experimental gas-to-IL and water-to-IL partition coefficient data to within average standard deviations of 0.100 and 0.133 log units, respectively.
\end{abstract}

\section{INTRODUCTION}

Next generation ionic liquids (ILs) have spawned considerable interest over the past decade owing to their unique physical properties and designer solvent qualities. Indeed, each year witnesses a considerable rise in the number of applied and issued patents centered around employing ILs as advanced solvent media. ILs are popular solvent choices for manufacturing applications involving nanomaterials and organic synthesis, as chromatographic stationary phases for chemical separations, as extraction solvent systems for the removal of aromatic nitrogen and sulfur compounds from coal and petroleum feedstocks, and more recently, as solvents for lignocellulosic biomass processing used to produce liquid fuels from agricultural wastes and wood feedstocks. Select alkylimidazoliumbased ILs have exhibited large selectivities for carbon dioxide over other gases, making them ideally suited for the removal and recovery of carbon dioxide from mixed gases. Saravanamyrugan et al. ${ }^{1}$ have further demonstrated that ILs can be tuned by design to perform as selective, high-density absorbents for removal of sulfur dioxide, nitric oxide, and/or carbon dioxide from industrial flue gases. Such selectivity is typically achieved by functionalization of the cation and/or anion. Presently, more than $10^{3}$ different ILs are commercially available, although the number of cation-anion combinations is of course staggeringly larger, in excess of $10^{14,2,3}$ Obviously, it is not feasible to experimentally study a large number of possible cation-anion combinations in any meaningful way. Consequently, the development of predictive methods is indispensible to navigate this vast chemical space and select the most appropriate IL for a given task.

This study is a continuation of our investigations regarding the thermodynamic properties of solutes dissolved in IL solvents. In previous papers, ${ }^{4-10}$ we have shown that the introduction of polar side chains within ions comprising the IL exerts pronounced influence over the interaction with solute species present in mixtures containing the IL. Short polar chains present in imidazolium-based ILs, for example, increased the selectivity toward mixtures containing \{alcohol + aliphatic\} or \{aromatic + aliphatic\} solutes. The present work is focused on the solvent character of four "unfunctionalized" (i.e., aliphatic) tetraalkylammonium-based ILs, methyl(tributyl)ammonium bis(trifluoromethylsulfonyl)imide, $\left[\mathrm{MB}_{3} \mathrm{AM}\right]^{+}\left[\mathrm{Tf}_{2} \mathrm{~N}\right]^{-}$, octyl(trimethyl)ammonium bis(trifluoromethylsulfonyl)imide, $\left[\mathrm{OM}_{3} \mathrm{AM}\right]^{+}\left[\mathrm{Tf}_{2} \mathrm{~N}\right]^{-}$, decyl(trimethyl)ammonium bis(trifluoromethylsulfonyl)imide, $\left[\mathrm{DM}_{3} \mathrm{AM}\right]^{+}\left[\mathrm{Tf}_{2} \mathrm{~N}\right]^{-}$, and tetraoctylammonium bis(trifluoromethylsulfonyl)imide, $\left[\mathrm{O}_{4} \mathrm{AM}\right]^{+}\left[\mathrm{Tf}_{2} \mathrm{~N}\right]^{-}$ (molecular structures for these four ILs are presented in Figure 1), to determine what role (if any) the distribution of alkyl chain lengths play in the $\mathrm{IL}$ solvent's ability to dissolve select classes of organic compounds. These experimental data provide a benchmark for strategic future studies involving functionalized tetraalkylammonium-based ILs sporting polar alkyl chain substituents.

The Abraham solvation parameter model provides a convenient mathematical means to quantify and compare the solubilizing character of different solvent media using terms that represent the various types of solute-solvent interactions. The magnitude of the different solute-solvent interactions depends upon the hydrogen-bonding, electron-withdrawing, electron-donating, and aromatic functional groups contained in the molecules. Mathematical correlations based on the Abraham general solvation parameter model for the gas-to-solvent,

\section{Received: June 26, 2011}

Accepted: August 3, 2011

Published: August 18, 2011 


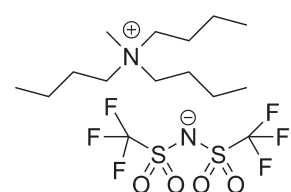

$\left[\mathrm{MB}_{3} \mathrm{Am}\right]^{+}\left[\mathrm{Tf}_{2} \mathrm{~N}\right]^{-}$

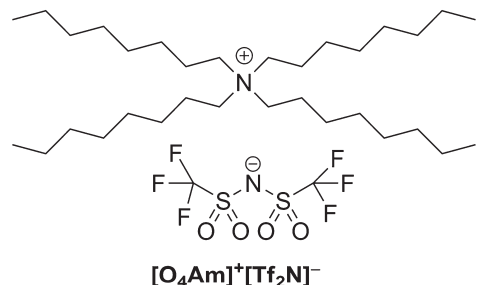

$\left[\mathrm{O}_{4} \mathrm{Am}\right]^{+}\left[\mathrm{Tf}_{2} \mathrm{~N}\right]^{-}$

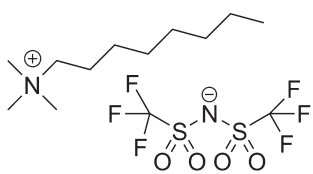

$\left[\mathrm{OM}_{3} \mathrm{Am}\right]^{+}\left[\mathrm{Tf}_{2} \mathrm{~N}\right]^{-}$

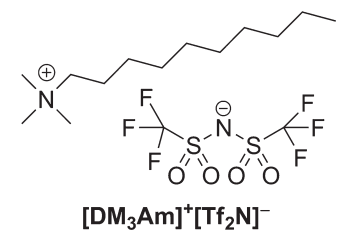

$\left.\mathrm{DM}_{3} \mathrm{Am}\right]^{+}\left[\mathrm{Tf}_{2} \mathrm{~N}\right]^{-}$
Figure 1. Chemical structures of the ILs scrutinized in this work.

$K_{\mathrm{L}}$, and water-to-solvent partition coefficients, $P$,

$$
\begin{aligned}
& \log P=c+e \cdot E+s \cdot S+a \cdot A+b \cdot B+v \cdot V \\
& \log K_{\mathrm{L}}=c+e \cdot E+s \cdot S+a \cdot A+b \cdot B+1 \cdot L
\end{aligned}
$$

have been reported for 26 different anhydrous ILs ${ }^{11-22}$ and for two practical water-to-IL partition systems. ${ }^{23}$ In eqs 1 and 2 , the independent variables (descriptors) are properties of the neutral solute as follows: $E$ is the solute excess molar refraction in $\mathrm{cm}^{3} \cdot \mathrm{mol}^{-1} / 10, S$ is the solute dipolarity/polarizability, $A$ and $B$ are the overall solute hydrogen bond acidity and basicity, $V$ is McGowan's characteristic molecular volume in units of $\mathrm{cm}^{3} \cdot \mathrm{mol}^{-1}$ / 100 , and $L$ is the logarithm of the gas-to-hexadecane partition coefficient at $298 \mathrm{~K}$. The regression coefficients and constants $(c, e, s, a, b, v$, and $l)$ are obtained by regression analysis of experimental data for a specific process (i.e., a given partitioning process/system). In the case of processes involving two condensed solvent phases, the $c, e, s, a, b, v$, and $l$ coefficients represent differences in the solvent phase properties. For any fully characterized system/process (those with calculated values for the equation coefficients), further values of $\log P$ and $\log K$ can be estimated with known values for the solute descriptors.

Partition coefficient correlations based on eqs 1 and 2 are specific to a given IL. Sprunger et al. ${ }^{24-26}$ modified the Abraham solvation parameter model

$$
\begin{aligned}
\log K_{\mathrm{L}}= & c_{\text {cation }}+c_{\text {anion }}+\left(e_{\text {cation }}+e_{\text {anion }}\right) E \\
& +\left(s_{\text {cation }}+s_{\text {anion }}\right) S+\left(a_{\text {cation }}+a_{\text {anion }}\right) A \\
& +\left(b_{\text {cation }}+b_{\text {anion }}\right) B+\left(l_{\text {cation }}+l_{\text {anion }}\right) L
\end{aligned}
$$

$$
\begin{aligned}
\log P= & c_{\text {cation }}+c_{\text {anion }}+\left(e_{\text {cation }}+e_{\text {anion }}\right) E \\
& +\left(s_{\text {cation }}+s_{\text {anion }}\right) S+\left(a_{\text {cation }}+a_{\text {anion }}\right) A \\
& +\left(b_{\text {cation }}+b_{\text {anion }}\right) B+\left(v_{\text {cation }}+v_{\text {anion }}\right) V
\end{aligned}
$$

by expressing each of the six solvent equation coefficients as a summation of their respective cation and anion contribution. In separating the equation coefficients, the authors make the assumption that a solute's interaction with a given cation is not influenced by the associated IL anion, and conversely that the solute's interaction with a given anion is not influenced by the surrounding IL cation. The major advantage derived from splitting the equation coefficients into individual cation- and anion-specific contributions is that it allows one to make predictions for additional ILs for which the cation- and anion-specific coeffcients are known. The 20 cation and 14 anion coefficients that have been calculated thus far were based on experimental data for 42 different ILs; ${ }^{20,22}$ these coefficients can, however, be combined to yield predictive expressions for far more ILs (i.e., $20 \times 14=$ $280 \mathrm{ILs}$ ). Results from the present study will enable the calculation of four additional sets of cation-specific equation coefficients and enable one to generate partition coefficient predictions for solutes dissolved in an additional 56 ILs.

\section{EXPERIMENTAL PROCEDURES AND RESULTS}

Materials or Chemicals. Decyl(trimethyl)ammonium bis(trifluoromethylsulfonyl)imide, $\left[\mathrm{DM}_{3} \mathrm{AM}\right]^{+}\left[\mathrm{Tf} \mathrm{N}_{2} \mathrm{~N}\right]^{-}$, was prepared to a purity exceeding $99.8 \%$ using methods reported previously. ${ }^{27,28}$ The synthesis of the remaining ILs methyl(tributyl)ammonium bis(trifluoromethylsulfonyl)imide, $\left[\mathrm{MB}_{3} \mathrm{AM}\right]^{+}$ $\left[\mathrm{Tf}_{2} \mathrm{~N}\right]^{-}$, octyl(trimethyl)ammonium bis(trifluoromethylsulfonyl)imide, $\left[\mathrm{OM}_{3} \mathrm{AM}\right]^{+}\left[\mathrm{Tf}_{2} \mathrm{~N}\right]^{-}$, and tetraoctylammonium bis(trifluoromethylsulfonyl)imide, $\left[\mathrm{O}_{4} \mathrm{AM}\right]^{+}\left[\mathrm{Tf}_{2} \mathrm{~N}\right]^{-}$, was performed similarly using twice-recrystallized bromide salts of the corresponding ammonium parents sourced from Sigma-Aldrich in $\geq 98 \%$ purity. In all cases, the materials obtained were clear, nearly colorless fluids with purities better than $99.8 \mathrm{wt} \%$ based on thermogravimetric analysis ${ }^{29}$ and verified by NMR spectroscopy.

Each IL was further purified by subjecting the liquid to a very low pressure of about $5 \mathrm{~Pa}$ at about $343 \mathrm{~K}$ for approximately $24 \mathrm{~h}$. Next, packed columns are conditioned during $12 \mathrm{~h}$. We assume that this procedure removed any volatile chemicals and water from the IL and Chromosorb. Beyond this soft thermal treatment, which has been shown to attain low water content in the (10 to 60) ppm range for a series of conventional imidazolium-, ammonium-, and pyrrolidinium-type $\mathrm{ILs},{ }^{30}$ no further attempt was made to analyze for impurities within the ILs. Densities and other physical properties of the ILs have been reported previously. ${ }^{27}$ Test solutes were purchased from Aldrich at a purity $\geq 99.5 \%$ and were used without further purification because our gas-liquid chromatography technique efficiently separates any impurities on the column.

Apparatus and Experimental Procedure. Inverse chromatography experiments were carried out using a Varian CP-3800 gas chromatograph equipped with a heated on-column injector and a flame ionization detector. The injector and detector temperatures were kept at $523 \mathrm{~K}$ during all experiments. The helium flow rate was adjusted to obtain adequate retention times. Methane was used to determine the column hold-up time. Exit gas flow rates were measured with an Alltech Digital Flow Check mass flowmeter. The temperature of the oven was measured with a Pt100 probe and controlled to within $0.1 \mathrm{~K}$. A personal computer directly recorded detector signals, and the corresponding chromatograms were obtained using Galaxie software.

Columns packed to $1 \mathrm{~m}$ lengths, containing from (15 to 35$) \%$ of stationary phases (IL) on Chromosorb WHP (60-80 mesh), were prepared using a rotary evaporation method. After evaporation of the chloroform in vacuo, the support was equilibrated at $333 \mathrm{~K}$ during $6 \mathrm{~h}$. Before measurement, each packed column was conditioned for $12 \mathrm{~h}$ at $363 \mathrm{~K}$ at a flow rate of $20 \mathrm{~cm}^{3} \cdot \mathrm{min}^{-1}$. The mass of the packing material was calculated from the mass of the packed and empty column and was checked during experiments. The masses of the stationary phase were determined with a precision of $\pm 0.0003 \mathrm{~g}$, which would correspond to less than a $0.1 \%$ relative uncertainty in the mass of the $\mathrm{IL}$ in the packed column. A volume of the headspace vapor of samples of (1 to 5) $\mu \mathrm{L}$ 
Table 1. Infinite Dilution Activity Coefficients and Partition Coefficients $\log K_{\mathrm{L}}$ and $\log P$ of Organic Solutes in $\left[\mathrm{MB}_{3} \mathrm{AM}\right]^{+}\left[\mathrm{Tf}_{2} \mathrm{~N}\right]^{-}$

\begin{tabular}{|c|c|c|c|c|c|}
\hline \multirow[b]{2}{*}{ solutes } & \multicolumn{3}{|c|}{$\gamma^{\infty}$ at $T / \mathrm{K}$} & $\log K_{\mathrm{L}}$ & \multirow[t]{2}{*}{$\log P$} \\
\hline & 322.85 & 332.65 & 343.15 & $298.15 \mathrm{~K}$ & \\
\hline hexane & 6.75 & 6.75 & 6.44 & 1.586 & 3.406 \\
\hline 3-methylpentane & 6.10 & 6.17 & 5.86 & 1.521 & 3.361 \\
\hline heptane & 8.88 & 8.82 & 8.34 & 1.984 & 3.944 \\
\hline 2,2,4-trimethylpentane & 8.87 & 8.91 & 8.42 & 1.964 & 4.084 \\
\hline octane & 11.57 & 11.49 & 10.80 & 2.363 & 4.473 \\
\hline nonane & 16.54 & 16.13 & 14.97 & 2.742 & 4.892 \\
\hline decane & 19.61 & 19.18 & 17.80 & 3.117 & 5.437 \\
\hline undecane & 25.74 & 24.81 & 22.80 & 3.484 & 5.864 \\
\hline dodecane & 32.35 & 31.31 & 28.79 & 3.782 & 6.312 \\
\hline methylcyclopentane & 4.75 & 4.74 & 4.50 & 1.806 & 2.976 \\
\hline cyclohexane & 4.74 & 4.71 & 4.44 & 1.959 & 2.859 \\
\hline methylcyclohexane & 5.92 & 5.91 & 5.58 & 2.178 & 3.428 \\
\hline cycloheptane & 12.40 & 17.74 & 23.93 & 2.502 & 3.092 \\
\hline benzene & 0.90 & 0.93 & 0.92 & 2.796 & 2.166 \\
\hline toluene & 1.13 & 1.19 & 1.19 & 3.196 & 2.546 \\
\hline ethylbenzene & 1.56 & 1.64 & 1.63 & 3.494 & 2.914 \\
\hline$m$-xylene & 1.49 & 1.56 & 1.56 & 3.573 & 2.963 \\
\hline$p$-xylene & 1.51 & 1.62 & 1.56 & 3.557 & 2.967 \\
\hline$o$-xylene & 1.38 & 1.46 & 1.46 & 3.724 & 2.984 \\
\hline 1-hexene & 4.31 & 4.40 & 4.25 & 1.704 & 2.864 \\
\hline 1-hexyne & 2.12 & 2.24 & 2.20 & 2.225 & 2.435 \\
\hline 1-heptyne & 2.79 & 2.89 & 2.85 & 2.619 & 3.059 \\
\hline 2-butanone & 0.40 & 0.38 & 0.35 & 3.025 & 0.305 \\
\hline 2-pentanone & 0.55 & 0.58 & 0.59 & 3.348 & 0.768 \\
\hline 3-pentanone & 0.50 & 0.54 & 0.56 & 3.354 & 0.854 \\
\hline 1,4-dioxane & 0.61 & 0.67 & 0.67 & 3.288 & -0.422 \\
\hline methanol & 0.96 & 0.75 & 0.88 & 2.357 & -1.383 \\
\hline ethanol & 1.53 & 1.40 & 1.27 & 2.565 & -1.105 \\
\hline 1-propanol & 1.78 & 1.64 & 1.48 & 2.960 & -0.600 \\
\hline 2-propanol & 1.67 & 1.54 & 1.40 & 2.660 & -0.820 \\
\hline 2-methyl-1-propanol & 1.97 & 1.82 & 1.64 & 3.170 & -0.130 \\
\hline 1-butanol & 2.10 & 1.89 & 1.73 & 3.364 & -0.096 \\
\hline trifluoroethanol & 0.50 & 0.48 & 0.45 & 3.124 & -0.036 \\
\hline diethyl ether & 1.65 & 1.67 & 1.65 & 1.681 & 0.511 \\
\hline diisopropyl ether & 3.31 & 3.35 & 3.35 & 1.929 & 0.879 \\
\hline chloroform & 0.84 & 0.87 & 0.88 & 2.577 & 1.787 \\
\hline dichloromethane & 0.66 & 0.69 & 0.70 & 2.179 & 1.219 \\
\hline tetrachloromethane & 2.11 & 2.11 & 2.07 & 2.367 & 2.557 \\
\hline acetonitrile & 0.49 & 0.50 & 0.48 & 2.956 & 0.106 \\
\hline nitromethane & 0.65 & 0.65 & 0.62 & 3.253 & 0.303 \\
\hline 1-nitropropane & 0.68 & 0.70 & 0.68 & 3.816 & 1.366 \\
\hline pyridine & 0.55 & 0.56 & 0.56 & 3.622 & 0.182 \\
\hline thiophene & 0.86 & 0.88 & 0.87 & 2.900 & 1.860 \\
\hline formaldehyde & 0.11 & 0.12 & 0.13 & 2.114 & \\
\hline propionaldehyde & 0.49 & 0.49 & 0.50 & 2.453 & -0.067 \\
\hline
\end{tabular}

was introduced to meet "infinite dilution" conditions. Each experiment was repeated at least twice to validate the reproducibility. Retention times were generally reproducible to within (0.01 to 0.03 ) $\mathrm{min}$. To check stability under our experimental conditions, such as the possible elution of the stationary phase by the helium stream, measurements of retention time were systematically repeated every day for three selected solutes. No changes in the retention times were observed during this stability study.

\section{THEORETICAL BASIS}

The retention data determined using inverse chromatography experiments were used to calculate partition coefficients for each solute in the IL. The net retention volume, $V_{\mathrm{N}}$, was calculated from the following relationship: ${ }^{31}$

$$
V_{\mathrm{N}}=\frac{3}{2} \cdot \frac{\left[\left(P_{\mathrm{i}} / P_{\mathrm{o}}\right)^{2}-1\right]}{\left[\left(P_{\mathrm{i}} / P_{\mathrm{o}}\right)^{3}-1\right]} U_{\mathrm{o}} \cdot t_{\mathrm{R}}^{\prime} \cdot \frac{T_{\mathrm{col}}}{T_{\mathrm{r}}} \cdot\left(1-\frac{P_{\mathrm{w}}^{0}}{P_{\mathrm{o}}}\right)
$$

The adjusted retention time $t_{\mathrm{R}}{ }^{\prime}$ was taken as a difference between the retention time of a solute and that of methane, $T_{\text {col }}$ is the column temperature, $U_{0}$ is the flow rate of the carrier gas measured at the room temperature $\left(T_{\mathrm{r}}\right)$, and $P_{\mathrm{w}}^{\mathrm{o}}$ is the vapor pressure of water at $T_{\mathrm{r}} . P_{\mathrm{i}}$ and $P_{0}$ are, respectively, the inlet and outlet pressures.

The activity coefficient at infinite dilution of solute " 1 " in the IL " 2 ", $\gamma_{1,2}^{\infty}$, was calculated with the following expression: ${ }^{31}$

$$
\ln \gamma_{1,2}^{\infty}=\ln \left(\frac{n_{2} R T}{V_{\mathrm{N}} P_{1}^{0}}\right)-P_{1}^{0} \cdot \frac{B_{11}-V_{1}^{0}}{R T}+\frac{2 B_{13}-V_{1}^{\infty}}{R T} \cdot J P_{0}
$$

Here, $n_{2}$ is the mole number of the stationary phase component inside the column, $R$ is the ideal gas constant, $T$ is the temperature of the oven, $B_{11}$ is the second virial coefficient of the solute in the gaseous state at temperature $T, B_{13}$ is the mutual virial coefficient between the solute (" 1 ") and the carrier gas helium (" 3 "), and $P_{1}{ }^{0}$ is the probe vapor pressure at temperature $T$.

Partition coefficients, $K_{\mathrm{L}}$, may be then calculated from the activity coefficients at infinite dilution, $\gamma_{1,2}^{\infty}$, using the following equation:

$$
K_{\mathrm{L}}=\frac{R T}{\gamma_{1,2}^{\infty} P_{1}^{0} V_{\text {solvent }}}
$$

Thermodynamic properties of pure solutes needed for these calculations can be found in a previous work. ${ }^{4}$

\section{RESULTS AND DISCUSSION}

The errors in the experimental determination of activity coefficient are evaluated to be about $3 \%$. For all ILs studied in this work, no interfacial adsorption was observed while the average relative standard deviation between data sets obtained from different packed columns was (3 to 4) \%. Experimental activity coefficients at infinite dilution and gas-to-IL partition coefficients calculated using eqs 3 to 7 are listed in Tables 1 to 8 . Most solutes were found to be more soluble in the tetraalkylammonium ILs than in dialkylimidazolium ILs. Activity coefficients at infinite dilution for most organic compounds decreased with an increase in temperature. Naturally, the solubility of apolar compounds increases with an increase in the alkyl chain length grafted onto the ammonium cation. For compounds containing the same number of carbon atoms but originating from different 
Table 2. Logarithm of the Gas-to-IL Partition Coefficients for Solutes Dissolved in $\left[\mathrm{MB}_{3} \mathrm{AM}\right]^{+}\left[\mathrm{Tf}_{2} \mathrm{~N}\right]^{-}$

\begin{tabular}{|c|c|c|c|c|c|}
\hline$T / \mathrm{K}$ & 322.85 & 332.65 & 343.15 & $R^{2}$ & 298.15 \\
\hline \multicolumn{6}{|l|}{ Solutes } \\
\hline hexane & 1.284 & 1.177 & 1.080 & 0.999 & 1.586 \\
\hline 3-methylpentane & 1.244 & 1.146 & 1.049 & 1.000 & 1.521 \\
\hline heptane & 1.617 & 1.486 & 1.368 & 0.999 & 1.984 \\
\hline 2,2,4-trimethylpentane & 1.611 & 1.486 & 1.368 & 1.000 & 1.964 \\
\hline octane & 1.943 & 1.794 & 1.656 & 0.999 & 2.363 \\
\hline nonane & 2.266 & 2.097 & 1.941 & 0.999 & 2.742 \\
\hline decane & 2.585 & 2.396 & 2.221 & 0.999 & 3.117 \\
\hline undecane & 2.899 & 2.692 & 2.499 & 0.999 & 3.484 \\
\hline dodecane & 3.213 & 2.984 & 2.774 & 0.999 & 3.782 \\
\hline methylcyclopentane & 1.497 & 1.388 & 1.286 & 0.999 & 1.806 \\
\hline cyclohexane & 1.640 & 1.526 & 1.418 & 1.000 & 1.959 \\
\hline methylcyclohexane & 1.826 & 1.701 & 1.582 & 1.000 & 2.178 \\
\hline cycloheptane & 2.116 & 1.979 & 1.850 & 1.000 & 2.502 \\
\hline benzene & 2.386 & 2.240 & 2.103 & 1.000 & 2.796 \\
\hline toluene & 2.728 & 2.561 & 2.405 & 1.000 & 3.196 \\
\hline ethylbenzene & 2.987 & 2.807 & 2.636 & 1.000 & 3.494 \\
\hline$m$-xylene & 3.051 & 2.865 & 2.689 & 1.000 & 3.573 \\
\hline$p$-xylene & 3.034 & 2.848 & 2.672 & 1.000 & 3.557 \\
\hline$o$-xylene & 3.185 & 2.993 & 2.815 & 1.000 & 3.724 \\
\hline 1-hexene & 1.397 & 1.288 & 1.182 & 1.000 & 1.704 \\
\hline 1-hexyne & 1.847 & 1.712 & 1.584 & 1.000 & 2.225 \\
\hline 1-heptyne & 2.183 & 2.028 & 1.881 & 1.000 & 2.619 \\
\hline 2-butanone & 2.596 & 2.443 & 2.295 & 1.000 & 3.025 \\
\hline 2-pentanone & 2.879 & 2.712 & 2.551 & 1.000 & 3.348 \\
\hline 3-pentanone & 2.878 & 2.709 & 2.546 & 1.000 & 3.354 \\
\hline 1,4-dioxane & 2.818 & 2.651 & 2.493 & 1.000 & 3.288 \\
\hline methanol & 2.101 & 2.010 & 1.827 & 0.965 & 2.357 \\
\hline ethanol & 2.174 & 2.035 & 1.896 & 1.000 & 2.565 \\
\hline 1-propanol & 2.496 & 2.331 & 2.178 & 0.999 & 2.960 \\
\hline 2-propanol & 2.226 & 2.072 & 1.928 & 1.000 & 2.660 \\
\hline 2-methyl-1-propanol & 2.672 & 2.495 & 2.331 & 0.999 & 3.170 \\
\hline 1-butanol & 2.850 & 2.668 & 2.496 & 1.000 & 3.364 \\
\hline trifluoroethanol & 2.653 & 2.486 & 2.329 & 1.000 & 3.124 \\
\hline diethyl ether & 1.374 & 1.265 & 1.157 & 1.000 & 1.681 \\
\hline diisopropyl ether & 1.570 & 1.442 & 1.317 & 1.000 & 1.929 \\
\hline chloroform & 2.170 & 2.025 & 1.889 & 1.000 & 2.577 \\
\hline dichloromethane & 1.845 & 1.727 & 1.613 & 1.000 & 2.179 \\
\hline tetrachloromethane & 1.980 & 1.842 & 1.712 & 1.000 & 2.367 \\
\hline acetonitrile & 2.601 & 2.475 & 2.346 & 1.000 & 2.956 \\
\hline nitromethane & 2.836 & 2.688 & 2.546 & 1.000 & 3.253 \\
\hline 1-nitropropane & 3.309 & 3.129 & 2.959 & 1.000 & 3.816 \\
\hline pyridine & 3.171 & 3.011 & 2.858 & 1.000 & 3.622 \\
\hline thiophene & 2.478 & 2.328 & 2.187 & 1.000 & 2.900 \\
\hline formaldehyde & 1.764 & 1.640 & 1.512 & 1.000 & 2.114 \\
\hline propionaldehyde & 2.090 & 1.961 & 1.834 & 1.000 & 2.453 \\
\hline butyraldehyde & 2.408 & 2.257 & 2.117 & 0.999 & 2.832 \\
\hline
\end{tabular}

solute families, it was observed that $\gamma_{\text {aromatic }}<\gamma_{\text {alkyne }}<\gamma_{\text {alkene }}<$ $\gamma_{\text {alkane. This overall trend is followed for all ILs, regardless of the }}$ cation or anion identity. The presence of double or triple bonds in a solute increases the solute-IL interactions. Generally, activity coefficients at infinite dilution of the alcohols are
Table 3. Infinite Dilution Activity Coefficients and Partition Coefficients $\log K_{\mathrm{L}}$ and $\log P$ of Organic Solutes in $\left[\mathrm{OM}_{3} \mathrm{AM}\right]^{+}\left[\mathrm{Tf}_{2} \mathbf{N}\right]^{-}$

\begin{tabular}{|c|c|c|c|c|c|}
\hline \multirow{2}{*}{ solutes } & \multicolumn{3}{|c|}{$\gamma^{\infty}$ at $T / K$} & \multirow{2}{*}{$\frac{\log K_{\mathrm{L}}}{298 .}$} & \multirow{2}{*}{$\frac{\log P}{5 \mathrm{~K}}$} \\
\hline & 322.85 & 332.65 & 343.15 & & \\
\hline hexane & 6.21 & 5.83 & 5.36 & 1.672 & 3.492 \\
\hline 3-methylpentane & 5.62 & 5.24 & 4.87 & 1.633 & 3.473 \\
\hline heptane & 8.27 & 7.81 & 7.18 & 2.060 & 4.020 \\
\hline 2,2,4-trimethylpentane & 8.10 & 7.66 & 7.14 & 2.046 & 4.166 \\
\hline octane & 11.00 & 10.32 & 9.47 & 2.458 & 4.568 \\
\hline nonane & 15.95 & 14.81 & 13.36 & 2.835 & 4.985 \\
\hline decane & 19.33 & 17.95 & 16.20 & 3.222 & 5.542 \\
\hline undecane & 25.83 & 23.68 & 21.10 & 3.592 & 5.972 \\
\hline dodecane & 33.12 & 30.51 & 27.15 & 3.973 & 6.503 \\
\hline methylcyclopentane & 4.18 & 3.95 & 3.67 & 1.870 & 3.040 \\
\hline cyclohexane & 4.08 & 3.84 & 3.57 & 2.010 & 2.910 \\
\hline methylcyclohexane & 5.23 & 4.93 & 4.60 & 2.238 & 3.488 \\
\hline cycloheptane & 10.83 & 14.65 & 19.42 & 2.561 & 3.141 \\
\hline benzene & 0.73 & 0.74 & 0.73 & 2.792 & 2.162 \\
\hline toluene & 0.97 & 0.98 & 0.98 & 3.226 & 2.576 \\
\hline ethylbenzene & 1.39 & 1.40 & 1.38 & 3.541 & 2.961 \\
\hline$m$-xylene & 1.39 & 1.39 & 1.38 & 3.646 & 3.036 \\
\hline$p$-xylene & 1.38 & 1.39 & 1.37 & 3.638 & 3.048 \\
\hline$o$-xylene & 1.24 & 1.26 & 1.25 & 3.779 & 3.119 \\
\hline 1-hexene & 3.99 & 3.80 & 3.60 & 1.808 & 2.968 \\
\hline 1-hexyne & 1.92 & 1.89 & 1.82 & 2.321 & 2.531 \\
\hline 1-heptyne & 2.48 & 2.46 & 2.39 & 2.668 & 3.118 \\
\hline 2-butanone & 0.41 & 0.38 & 0.34 & 3.151 & 0.431 \\
\hline 2-pentanone & 0.57 & 0.57 & 0.57 & 3.481 & 0.901 \\
\hline 3-pentanone & 0.52 & 0.53 & 0.54 & 3.495 & 0.995 \\
\hline 1,4-dioxane & 0.62 & 0.62 & 0.61 & 3.484 & -0.226 \\
\hline methanol & 0.92 & 0.78 & 0.81 & 2.289 & -1.451 \\
\hline ethanol & 1.47 & 1.32 & 1.19 & 2.620 & -1.050 \\
\hline 1-propanol & 1.70 & 1.55 & 1.37 & 3.008 & -0.552 \\
\hline 2-propanol & 1.63 & 1.48 & 1.32 & 2.720 & -0.760 \\
\hline 2-methyl-1-propanol & 1.87 & 1.70 & 1.51 & 3.218 & -0.082 \\
\hline 1-butanol & 2.01 & 1.80 & 1.59 & 3.399 & -0.061 \\
\hline trifluoroethanol & 0.40 & 0.38 & 0.35 & 3.086 & -0.074 \\
\hline diethyl ether & 1.60 & 1.57 & 1.53 & 1.757 & 0.587 \\
\hline diisopropyl ether & 3.22 & 3.15 & 3.08 & 2.000 & 0.950 \\
\hline chloroform & 0.63 & 0.65 & 0.66 & 2.508 & 1.718 \\
\hline dichloromethane & 0.51 & 0.53 & 0.54 & $2.129^{a}$ & $1.169^{a}$ \\
\hline tetrachloromethane & 1.63 & 1.63 & 1.60 & 2.302 & 2.492 \\
\hline acetonitrile & 0.48 & 0.46 & 0.44 & 3.079 & 0.229 \\
\hline nitromethane & 0.60 & 0.58 & 0.55 & 3.320 & 0.370 \\
\hline 1-nitropropane & 0.62 & 0.61 & 0.60 & 3.873 & 1.423 \\
\hline pyridine & 0.45 & 0.44 & 0.43 & 3.629 & 0.189 \\
\hline thiophene & 0.69 & 0.69 & 0.69 & 2.860 & 1.820 \\
\hline formaldehyde & 0.10 & 0.11 & 0.12 & 2.191 & \\
\hline propionaldehyde & 0.48 & 0.47 & 0.46 & 2.498 & -0.022 \\
\hline
\end{tabular}

relatively small, with the solubility of alcohols and chloroalkanes being higher in $\left[\mathrm{O}_{4} \mathrm{Am}\right]^{+}\left[\mathrm{Tf}_{2} \mathrm{~N}\right]^{-}$than in the other tetraalkylammonium studied in this work. The hydroxyl group 
Table 4. Logarithm of the Gas-to-IL Partition Coefficients for Solutes Dissolved in $\left[\mathrm{OM}_{3} \mathrm{AM}\right]^{+}\left[\mathrm{Tf}_{2} \mathrm{~N}\right]^{-}$

\begin{tabular}{|c|c|c|c|c|c|}
\hline$T / \mathrm{K}$ & 322.85 & 332.65 & 343.15 & $R^{2}$ & 298.15 \\
\hline \multicolumn{6}{|l|}{ Solutes } \\
\hline hexane & 1.295 & 1.161 & 1.048 & 0.997 & 1.672 \\
\hline 3-methylpentane & 1.256 & 1.122 & 1.016 & 0.995 & 1.633 \\
\hline heptane & 1.633 & 1.481 & 1.351 & 0.998 & 2.060 \\
\hline 2,2,4-trimethylpentane & 1.619 & 1.467 & 1.343 & 0.996 & 2.046 \\
\hline octane & 1.969 & 1.795 & 1.646 & 0.998 & 2.458 \\
\hline nonane & 2.298 & 2.107 & 1.939 & 0.999 & 2.835 \\
\hline decane & 2.626 & 2.414 & 2.228 & 0.999 & 3.222 \\
\hline undecane & 2.948 & 2.719 & 2.512 & 0.999 & 3.592 \\
\hline dodecane & 3.270 & 3.020 & 2.795 & 0.999 & 3.973 \\
\hline methylcyclopentane & 1.490 & 1.355 & 1.244 & 0.997 & 1.870 \\
\hline cyclohexane & 1.622 & 1.484 & 1.371 & 0.997 & 2.010 \\
\hline methylcyclohexane & 1.819 & 1.670 & 1.545 & 0.997 & 2.238 \\
\hline cycloheptane & 2.105 & 1.943 & 1.807 & 0.997 & 2.561 \\
\hline benzene & 2.345 & 2.186 & 2.047 & 0.998 & 2.792 \\
\hline toluene & 2.711 & 2.528 & 2.368 & 0.998 & 3.226 \\
\hline ethylbenzene & 2.984 & 2.786 & 2.611 & 0.999 & 3.541 \\
\hline$m$-xylene & 3.067 & 2.861 & 2.682 & 0.998 & 3.646 \\
\hline$p$-xylene & 3.042 & 2.830 & 2.663 & 0.995 & 3.638 \\
\hline$o$-xylene & 3.188 & 2.978 & 2.795 & 0.998 & 3.779 \\
\hline 1-hexene & 1.412 & 1.271 & 1.158 & 0.996 & 1.808 \\
\hline 1-hexyne & 1.851 & 1.684 & 1.550 & 0.996 & 2.321 \\
\hline 1-heptyne & 2.179 & 2.005 & 1.852 & 0.998 & 2.668 \\
\hline 2-butanone & 2.659 & 2.484 & 2.327 & 0.999 & 3.151 \\
\hline 2-pentanone & 2.944 & 2.753 & 2.585 & 0.999 & 3.481 \\
\hline 3-pentanone & 2.944 & 2.748 & 2.578 & 0.998 & 3.495 \\
\hline 1,4-dioxane & 2.879 & 2.664 & 2.500 & 0.994 & 3.484 \\
\hline methanol & 2.129 & 2.072 & 1.836 & 0.890 & 2.289 \\
\hline ethanol & 2.204 & 2.056 & 1.917 & 1.000 & 2.620 \\
\hline 1-propanol & 2.524 & 2.352 & 2.190 & 1.000 & 3.008 \\
\hline 2-propanol & 2.264 & 2.102 & 1.951 & 0.999 & 2.720 \\
\hline 2-methyl-1-propanol & 2.698 & 2.513 & 2.343 & 0.999 & 3.218 \\
\hline 1-butanol & 2.879 & 2.694 & 2.507 & 1.000 & 3.399 \\
\hline trifluoroethanol & 2.602 & 2.430 & 2.272 & 0.999 & 3.086 \\
\hline diethyl ether & 1.408 & 1.284 & 1.172 & 0.999 & 1.757 \\
\hline diisopropyl ether & 1.604 & 1.463 & 1.328 & 1.000 & 2.000 \\
\hline chloroform & 2.092 & 1.944 & 1.808 & 0.999 & 2.508 \\
\hline dichloromethane & 1.786 & 1.664 & 1.547 & 1.000 & 2.129 \\
\hline tetrachloromethane & 1.914 & 1.776 & 1.648 & 0.999 & 2.302 \\
\hline acetonitrile & 2.640 & 2.484 & 2.352 & 0.998 & 3.079 \\
\hline nitromethane & 2.853 & 2.687 & 2.544 & 0.998 & 3.320 \\
\hline 1-nitropropane & 3.319 & 3.122 & 2.948 & 0.999 & 3.873 \\
\hline triethylamine & 1.923 & 1.799 & 1.569 & 0.972 & 2.272 \\
\hline pyridine & 3.134 & 2.958 & 2.783 & 1.000 & 3.629 \\
\hline thiophene & 2.427 & 2.273 & 2.131 & 0.999 & 2.860 \\
\hline formaldehyde & 1.797 & 1.657 & 1.524 & 1.000 & 2.191 \\
\hline propionaldehyde & 2.124 & 1.991 & 1.852 & 1.000 & 2.498 \\
\hline
\end{tabular}

can potentially interact with either the anion and/or the cation of the IL. Interestingly, these classes of compound follow similar trends as the hydrocarbons. Branched-chain alcohols have a lower solubility compared to linear alcohols, and their solubility decreases with increasing chain length. The presence
Table 5. Infinite Dilution Activity Coefficients and Partition Coefficients $\log K_{\mathrm{L}}$ and $\log P$ of Organic Solutes at in $\left[\mathrm{DM}_{3} \mathrm{AM}\right]^{+}\left[\mathrm{Tf}_{2} \mathbf{N}\right]^{-}$

\begin{tabular}{|c|c|c|c|c|c|}
\hline \multirow{3}{*}{$\begin{array}{l}\text { solutes } \\
\text { hexane }\end{array}$} & \multicolumn{3}{|c|}{$\gamma^{\infty}$ at $T / K$} & \multirow{2}{*}{$\frac{\log K_{\mathrm{L}}}{298.1}$} & \multirow{2}{*}{$\frac{\log P}{\mathrm{~K}}$} \\
\hline & 322.85 & 332.65 & 343.15 & & \\
\hline & 4.20 & 4.10 & 3.93 & 1.783 & 3.603 \\
\hline 3-methylpentane & 3.88 & 3.78 & 3.62 & 1.721 & 3.561 \\
\hline heptane & 5.26 & 5.14 & 4.92 & 2.207 & 4.167 \\
\hline 2,2,4-trimethylpentane & 5.41 & 5.29 & 4.92 & 2.164 & 4.284 \\
\hline octane & 6.53 & 6.41 & 6.10 & 2.629 & 4.739 \\
\hline nonane & 8.87 & 8.63 & 7.61 & 3.035 & 5.185 \\
\hline decane & 10.07 & 9.82 & 9.32 & 3.445 & 5.765 \\
\hline undecane & 12.45 & 12.19 & 11.41 & 3.866 & 6.246 \\
\hline dodecane & 15.13 & 14.77 & 13.77 & 4.247 & 6.777 \\
\hline methylcyclopentane & 2.99 & 2.99 & 2.83 & 2.009 & 3.179 \\
\hline cyclohexane & 2.90 & 2.96 & 2.80 & 2.191 & 3.091 \\
\hline methylcyclohexane & 3.57 & 3.57 & 3.39 & 2.397 & 3.647 \\
\hline cycloheptane & 7.70 & 10.76 & 14.62 & 2.675 & 3.255 \\
\hline benzene & 0.71 & 0.73 & 0.72 & 2.803 & 2.173 \\
\hline toluene & 0.84 & 0.89 & 0.89 & 3.307 & 2.657 \\
\hline ethylbenzene & 1.16 & 1.20 & 1.19 & 3.590 & 3.010 \\
\hline$m$-xylene & 1.11 & 1.14 & 1.11 & 3.685 & 3.075 \\
\hline$p$-xylene & 1.12 & 1.15 & 1.13 & 3.664 & 3.074 \\
\hline$o$-xylene & 1.03 & 1.07 & 1.07 & 3.817 & 3.157 \\
\hline 1-hexene & 2.70 & 2.86 & 2.76 & 1.986 & 3.146 \\
\hline 1-hexyne & 1.61 & 1.63 & 1.57 & 2.324 & 2.534 \\
\hline 1-heptyne & 1.95 & 2.02 & 1.97 & 2.756 & 3.196 \\
\hline 2-butanone & 0.33 & 0.32 & 0.29 & 3.136 & 0.416 \\
\hline 2-pentanone & 0.44 & 0.47 & 0.47 & 3.490 & 0.910 \\
\hline 3-pentanone & 0.41 & 0.43 & 0.44 & 3.480 & 0.980 \\
\hline 1,4-dioxane & 0.56 & 0.57 & 0.57 & 3.346 & -0.364 \\
\hline methanol & 0.79 & 0.91 & 0.66 & 2.509 & -1.231 \\
\hline ethanol & 1.29 & 1.19 & 1.07 & 2.638 & -1.032 \\
\hline 1-propanol & 1.48 & 1.37 & 1.21 & 3.029 & -0.531 \\
\hline 2-propanol & 1.38 & 1.28 & 1.15 & 2.743 & -0.737 \\
\hline 2-methyl-1-propanol & 1.59 & 1.47 & 1.31 & 3.247 & -0.053 \\
\hline 1-butanol & 1.70 & 1.55 & 1.38 & 3.448 & -0.012 \\
\hline trifluoroethanol & 0.46 & 0.44 & 0.41 & 3.059 & -0.101 \\
\hline diethyl ether & 1.23 & 1.24 & 1.21 & 1.809 & 0.639 \\
\hline diisopropyl ether & 2.19 & 2.32 & 2.30 & 2.176 & 1.126 \\
\hline chloroform & 0.65 & 0.68 & 0.68 & 2.573 & 1.783 \\
\hline dichloromethane & 0.51 & 0.55 & 0.56 & 2.210 & 1.250 \\
\hline tetrachloromethane & 1.46 & 1.50 & 1.47 & 2.438 & 2.628 \\
\hline acetonitrile & 0.44 & 0.44 & 0.42 & 3.017 & 0.167 \\
\hline nitromethane & 0.56 & 0.58 & 0.55 & 3.356 & 0.406 \\
\hline 1-nitropropane & 0.59 & 0.59 & 0.57 & 3.843 & 1.393 \\
\hline triethylamine & 2.17 & 2.40 & 2.31 & 2.585 & 0.225 \\
\hline pyridine & 0.46 & 0.47 & 0.47 & 3.660 & 0.220 \\
\hline thiophene & 0.65 & 0.70 & 0.68 & 2.973 & 1.933 \\
\hline formaldehyde & 0.09 & 0.10 & 0.11 & 2.393 & \\
\hline propionaldehyde & 0.40 & 0.42 & 0.41 & 2.584 & 0.064 \\
\hline butyraldehyde & 0.47 & 0.53 & 0.51 & 3.051 & 0.721 \\
\hline
\end{tabular}

of a carboxyl group in a solute substantially increases its solubility in these ILs. 
Table 6. Logarithm of the Gas-to-IL Partition Coefficients for Solutes Dissolved in $\left[\mathrm{DM}_{3} \mathrm{AM}\right]^{+}\left[\mathrm{Tf}_{2} \mathrm{~N}\right]^{-}$

\begin{tabular}{|c|c|c|c|c|c|}
\hline$T / \mathrm{K}$ & 322.85 & 332.65 & 343.15 & $R^{2}$ & 298.15 \\
\hline \multicolumn{6}{|l|}{ Solutes } \\
\hline hexane & 1.437 & 1.314 & 1.199 & 0.999 & 1.783 \\
\hline 3-methylpentane & 1.389 & 1.271 & 1.161 & 1.000 & 1.721 \\
\hline heptane & 1.797 & 1.651 & 1.516 & 0.999 & 2.207 \\
\hline 2,2,4-trimethylpentane & 1.770 & 1.630 & 1.513 & 0.997 & 2.164 \\
\hline octane & 2.154 & 1.985 & 1.831 & 0.999 & 2.629 \\
\hline nonane & 2.504 & 2.315 & 2.169 & 0.994 & 3.035 \\
\hline decane & 2.852 & 2.641 & 2.445 & 0.999 & 3.445 \\
\hline undecane & 3.200 & 2.963 & 2.749 & 0.999 & 3.866 \\
\hline dodecane & 3.536 & 3.283 & 3.052 & 0.999 & 4.247 \\
\hline methylcyclopentane & 1.627 & 1.491 & 1.382 & 0.996 & 2.009 \\
\hline cyclohexane & 1.772 & 1.623 & 1.507 & 0.994 & 2.191 \\
\hline methylcyclohexane & 1.975 & 1.825 & 1.698 & 0.997 & 2.397 \\
\hline cycloheptane & 2.248 & 2.096 & 1.957 & 0.999 & 2.675 \\
\hline benzene & 2.381 & 2.231 & 2.095 & 0.999 & 2.803 \\
\hline toluene & 2.776 & 2.587 & 2.429 & 0.997 & 3.307 \\
\hline ethylbenzene & 3.050 & 2.858 & 2.685 & 0.999 & 3.590 \\
\hline$m$-xylene & 3.131 & 2.934 & 2.765 & 0.998 & 3.685 \\
\hline$p$-xylene & 3.110 & 2.913 & 2.737 & 0.999 & 3.664 \\
\hline$o$-xylene & 3.249 & 3.047 & 2.864 & 0.999 & 3.817 \\
\hline 1-hexene & 1.550 & 1.395 & 1.281 & 0.992 & 1.986 \\
\hline 1-hexyne & 1.908 & 1.760 & 1.632 & 0.998 & 2.324 \\
\hline 1-heptyne & 2.270 & 2.097 & 1.949 & 0.998 & 2.756 \\
\hline 2-butanone & 2.669 & 2.503 & 2.350 & 0.999 & 3.136 \\
\hline 2-pentanone & 2.970 & 2.785 & 2.618 & 0.999 & 3.490 \\
\hline 3-pentanone & 2.965 & 2.782 & 2.613 & 0.999 & 3.480 \\
\hline 1,4-dioxane & 2.848 & 2.671 & 2.510 & 0.999 & 3.346 \\
\hline methanol & 2.152 & 1.923 & 1.897 & 0.824 & 2.509 \\
\hline ethanol & 2.213 & 2.062 & 1.925 & 0.999 & 2.638 \\
\hline 1-propanol & 2.540 & 2.366 & 2.214 & 0.998 & 3.029 \\
\hline 2-propanol & 2.282 & 2.118 & 1.972 & 0.999 & 2.743 \\
\hline 2-methyl-1-propanol & 2.727 & 2.542 & 2.374 & 0.999 & 3.247 \\
\hline 1-butanol & 2.908 & 2.716 & 2.541 & 0.999 & 3.448 \\
\hline trifluoroethanol & 2.578 & 2.407 & 2.253 & 0.999 & 3.059 \\
\hline diethyl ether & 1.472 & 1.352 & 1.242 & 0.999 & 1.809 \\
\hline diisopropyl ether & 1.720 & 1.558 & 1.428 & 0.996 & 2.176 \\
\hline chloroform & 2.140 & 1.986 & 1.854 & 0.998 & 2.573 \\
\hline dichloromethane & 1.830 & 1.695 & 1.582 & 0.997 & 2.210 \\
\hline tetrachloromethane & 2.011 & 1.859 & 1.732 & 0.997 & 2.438 \\
\hline acetonitrile & 2.618 & 2.476 & 2.350 & 0.999 & 3.017 \\
\hline nitromethane & 2.853 & 2.674 & 2.529 & 0.996 & 3.356 \\
\hline 1-nitropropane & 3.317 & 3.130 & 2.961 & 0.999 & 3.843 \\
\hline triethylamine & 2.039 & 1.845 & 1.711 & 0.989 & 2.585 \\
\hline pyridine & 3.148 & 2.966 & 2.802 & 0.999 & 3.660 \\
\hline thiophene & 2.484 & 2.310 & 2.173 & 0.995 & 2.973 \\
\hline formaldehyde & 1.828 & 1.677 & 1.548 & 0.998 & 2.393 \\
\hline propionaldehyde & 2.151 & 1.997 & 1.873 & 0.996 & 2.584 \\
\hline butyraldehyde & 2.500 & 2.304 & 2.170 & 0.988 & 3.051 \\
\hline
\end{tabular}

The measurements conducted during this research offer an important means to assess the suitability of these tetraalkylammonium ILs as solvents in various separations. This is achieved through the calculation of the selectivity, $S_{12}^{\infty}$, and capacity, $k_{1}^{\infty}$, values at infinite dilution:

$$
\begin{aligned}
& S_{12}^{\infty}=\frac{\gamma_{1 / \mathrm{RTIL}}^{\infty}}{\gamma_{2 / \mathrm{RTIL}}^{\infty}} \\
& k_{1}^{\infty}=\frac{1}{\gamma_{1 / \mathrm{RTIL}}^{\infty}}
\end{aligned}
$$

In eq $8 \gamma_{1 / \text { RTIL }}^{\infty}$ and $\gamma_{2 / \text { RTIL }}^{\infty}$ denote the infinite dilution coefficients of the solutes 1 and 2 in the IL solvent. Selectivities and capacities at infinite dilution are reported in Table 9 for four separation problems at $323.15 \mathrm{~K}$ : hexane/benzene, hexane/ methanol, hexane/thiophene, and cyclohexane/thiophene. With regard to aliphatic/aromatic separations, these results indicate that tetralkylammanium are poor choices compared to imidazolium ILs. The selectivities obtained using ammonium-based ILs (between 3 and 9) are lower than classical solvents used industrially such as sulfolane $\left(S_{\text {hexane/benzene }}=30.5\right)$, dimethylsulfoxide $\left(S_{\text {hexane/benzene }}=22.7\right)$, and $N$-methyl-2-pyrrolidinone $\left(S_{\text {hexane/benzene }}=12.5\right) .{ }^{38}$ Nevertheless, a better capacity and selectivity may be obtained by a moderate lengthening in the alkyl chain grafted to the ammonium cation. Indeed, long alkyl chains grafted on the cation are known to increase the capacity, while being detrimental to selectivity. Although the results presented for these specific tetraalkylammonium ILs do not suggest that they will not attract particular interest for these separation problems, that it not to say that they will not be ideal for others.

Linear Solvation Energy Relationship (LSER) Characterization. The experimental data measured here can be used to calculate the Abraham model ion-specific equation coefficients for the methyl(tributyl)ammonium, octyl(trimethyl)ammonium, decyl(trimethyl)ammonium, and tetraoctylammonium cations for both the gas-to-IL (see eq 1) and water-to-IL (see eq 2) partitioning processes. The $\log P$ values for partition from water to the IL are calculated through eq 10

$$
\log P=\log K_{\mathrm{L}}-\log K_{\mathrm{W}}
$$

and require knowledge of the solute's gas phase partition coefficient into water, $K_{\mathrm{W}}$, which is available for most of the solutes studied. ${ }^{11,12}$ Water-to-IL partition coefficients calculated through eq 8 pertain to a hypothetical partitioning process involving solute transfer from water to the anhydrous $I L$. $\log P$ values calculated in this manner are still quite useful in that predicted $\log P$ values can be used to estimate the solute's infinite dilution activity coefficient in the IL. For convenience, we have tabulated in the last two columns of Tables 1 , 3, 5, and 7 the $\log K_{\mathrm{L}}$ and $\log P$ values at $298.15 \mathrm{~K}$ for anhydrous $\left[\mathrm{MB}_{3} \mathrm{AM}\right]^{+}\left[\mathrm{Tf}_{2} \mathrm{~N}\right]^{-}$and $\left[\mathrm{OM}_{3} \mathrm{AM}\right]^{+}\left[\mathrm{Tf}_{2} \mathrm{~N}\right]^{-}$and $\left[\mathrm{DM}_{3} \mathrm{AM}\right]^{+}$ $\left[\mathrm{Tf}_{2} \mathrm{~N}\right]^{-}$and $\left[\mathrm{O}_{4} \mathrm{AM}\right]^{+}\left[\mathrm{Tf}_{2} \mathrm{~N}\right]^{-}$, respectively. The numerical values of $\log K_{\mathrm{L}}$ at 298 were obtained by extrapolating the measured values at higher temperatures assuming a $\log K_{\mathrm{L}}$ versus $1 / T$ linear relationship.

The 44 experimental $\log K_{\mathrm{L}}$ and $\log P$ data in Table 1 for $\left[\mathrm{MB}_{3} \mathrm{AM}\right]^{+}\left[\mathrm{Tf}_{2} \mathrm{~N}\right]^{-}$were analyzed in accordance with eqs 1 and 2 of the Abraham model. The calculated equation coefficients and associated statistical information are given in Table 10, where $N$ denotes the number of experimental values used in the regression analysis; SD refers to the standard deviation; $R^{2}$ is the squared correlation coefficient; and $F$ corresponds to the Fisher F-statistic. The standard errors in the calculated coefficients are given in parentheses immediately below the respective value. The $e \cdot E$ term 
Table 7. Infinite Dilution Activity Coefficients and Partition Coefficients $\log K_{\mathrm{L}}$ and $\log P$ of Organic Solutes in $\left[\mathrm{O}_{4} \mathrm{AM}\right]^{+}\left[\mathrm{Tf}_{2} \mathbf{N}\right]^{-}$

\begin{tabular}{|c|c|c|c|c|c|}
\hline \multirow{3}{*}{$\begin{array}{l}\text { solutes } \\
\text { hexane }\end{array}$} & \multicolumn{3}{|c|}{$\gamma^{\infty}$ at $T / \mathrm{K}$} & \multirow{2}{*}{$\frac{\log K_{\mathrm{L}}}{298.1}$} & \multirow{2}{*}{$\frac{\log P}{\mathrm{~K}}$} \\
\hline & 323.15 & 333.15 & 343.15 & & \\
\hline & 1.30 & 1.29 & 1.28 & 2.165 & 3.985 \\
\hline 3-methylpentane & 1.22 & 1.22 & 1.21 & 2.098 & 3.938 \\
\hline heptane & 1.50 & 1.48 & 1.47 & 2.620 & 4.580 \\
\hline 2,2,4-trimethylpentane & 1.52 & 1.51 & 1.50 & 2.587 & 4.707 \\
\hline octane & 1.73 & 1.71 & 1.69 & 3.066 & 5.176 \\
\hline nonane & 2.19 & 2.14 & 2.09 & 3.503 & 5.653 \\
\hline decane & 2.30 & 2.32 & 2.21 & 3.937 & 6.257 \\
\hline undecane & & & 2.54 & & \\
\hline dodecane & & & 2.72 & & \\
\hline methylcyclopentane & 0.98 & 0.97 & 0.97 & 2.331 & 3.501 \\
\hline cyclohexane & 0.95 & 0.94 & 0.92 & 2.483 & 3.383 \\
\hline methylcyclohexane & 1.07 & 1.06 & 1.05 & 2.761 & 4.011 \\
\hline cycloheptane & 2.21 & 3.16 & 4.37 & 3.088 & 3.678 \\
\hline benzene & 0.40 & 0.41 & 0.41 & 2.914 & 2.284 \\
\hline toluene & 0.47 & 0.48 & 0.49 & 3.370 & 2.720 \\
\hline ethylbenzene & 0.59 & 0.60 & 0.61 & 3.715 & 3.135 \\
\hline$m$-xylene & & 0.60 & 0.62 & 3.802 & 3.192 \\
\hline$p$-xylene & & 0.60 & 0.62 & 3.779 & 3.189 \\
\hline$o$-xylene & & 0.57 & 0.58 & 3.906 & 3.166 \\
\hline 1-hexene & 1.05 & 1.05 & 1.04 & 2.178 & 3.338 \\
\hline 1-hexyne & 0.75 & 0.76 & 0.76 & 2.506 & 2.716 \\
\hline 1-heptyne & 0.84 & 0.86 & 0.87 & 2.963 & 3.403 \\
\hline 2-butanone & 0.29 & 0.28 & 0.26 & 3.032 & 0.312 \\
\hline 2-pentanone & 0.35 & 0.36 & 0.37 & 3.432 & 0.852 \\
\hline 3-pentanone & 0.32 & 0.33 & 0.35 & 3.423 & 0.923 \\
\hline 1,4-dioxane & 0.47 & 0.47 & 0.47 & 3.263 & -0.447 \\
\hline methanol & 0.65 & 0.76 & 0.82 & 2.692 & -1.048 \\
\hline ethanol & 1.18 & 1.12 & 1.05 & 2.553 & -1.117 \\
\hline 1-propanol & 1.22 & 1.14 & 1.04 & 2.959 & -0.601 \\
\hline 2-propanol & 1.21 & 1.13 & 1.05 & 2.651 & -0.829 \\
\hline 2-methyl-1-propanol & 1.17 & 1.09 & 1.00 & 3.226 & -0.074 \\
\hline trifluoroethanol & 0.48 & 0.37 & 0.36 & 2.761 & -0.399 \\
\hline diethyl ether & 0.71 & 0.71 & 0.71 & 1.898 & 0.728 \\
\hline diisopropyl ether & 1.08 & 1.08 & 1.08 & 2.268 & 1.218 \\
\hline chloroform & 0.34 & 0.35 & 0.36 & 2.685 & 1.895 \\
\hline dichloromethane & 0.29 & 0.30 & 0.31 & 2.281 & 1.321 \\
\hline tetrachloromethane & 0.63 & 0.64 & 0.64 & 2.625 & 2.815 \\
\hline acetonitrile & 0.50 & 0.49 & 0.49 & 2.818 & -0.032 \\
\hline nitromethane & 0.63 & 0.61 & 0.59 & 3.073 & 0.123 \\
\hline 1-nitropropane & 0.48 & 0.48 & 0.47 & 3.769 & 1.319 \\
\hline triethylamine & 1.03 & 1.05 & 1.02 & 2.631 & 0.270 \\
\hline pyridine & 0.33 & 0.33 & 0.34 & 3.644 & 0.204 \\
\hline thiophene & 0.40 & 0.40 & 0.41 & 2.985 & 1.945 \\
\hline formaldehyde & 0.07 & 0.08 & 0.09 & 2.118 & \\
\hline propionaldehyde & 0.25 & 0.35 & 0.35 & 2.783 & 0.260 \\
\hline butyraldehyde & & & 0.31 & & \\
\hline
\end{tabular}

was found to negligible in the $\log P$ correlation $(e=-0.023)$ so it was removed from the final derived correlation. Solute descriptors used in the analysis are listed in Table 11 . Both equations provide a very
Table 8. Logarithm of the Gas-to-IL Partition Coefficients for Solutes Dissolved in $\left[\mathrm{O}_{4} \mathrm{AM}\right]^{+}\left[\mathrm{Tf}_{2} \mathrm{~N}\right]^{-}$

\begin{tabular}{|c|c|c|c|c|c|}
\hline$T / \mathrm{K}$ & 323.15 & 333.15 & 343.15 & $R^{2}$ & $298.15 \mathrm{~K}$ \\
\hline \multicolumn{6}{|l|}{ Solutes } \\
\hline hexane & 1.798 & 1.667 & 1.543 & 1.000 & 2.165 \\
\hline 3-methylpentane & 1.742 & 1.613 & 1.495 & 1.000 & 2.098 \\
\hline heptane & 2.194 & 2.040 & 1.898 & 1.000 & 2.620 \\
\hline 2,2,4-trimethylpentane & 2.173 & 2.025 & 1.886 & 1.000 & 2.587 \\
\hline octane & 2.582 & 2.407 & 2.245 & 1.000 & 3.066 \\
\hline nonane & 2.963 & 2.768 & 2.587 & 1.000 & 3.503 \\
\hline decane & 3.342 & 3.114 & 2.926 & 0.998 & 3.937 \\
\hline undecane & & & 3.259 & & \\
\hline dodecane & & & 3.613 & & \\
\hline methylcyclopentane & 1.962 & 1.831 & 1.706 & 1.000 & 2.331 \\
\hline cyclohexane & 2.108 & 1.972 & 1.847 & 1.000 & 2.483 \\
\hline methylcyclohexane & 2.350 & 2.202 & 2.064 & 1.000 & 2.761 \\
\hline cycloheptane & 2.646 & 2.485 & 2.338 & 1.000 & 3.088 \\
\hline benzene & 2.486 & 2.334 & 2.190 & 1.000 & 2.914 \\
\hline toluene & 2.883 & 2.708 & 2.544 & 1.000 & 3.370 \\
\hline ethylbenzene & 3.191 & 3.006 & 2.828 & 1.000 & 3.715 \\
\hline$m$-xylene & & 3.061 & 2.877 & 1.000 & 3.802 \\
\hline$p$-xylene & & 3.042 & 2.859 & 1.000 & 3.779 \\
\hline$o$-xylene & & 3.169 & 2.986 & 1.000 & 3.906 \\
\hline 1-hexene & 1.813 & 1.682 & 1.560 & 1.000 & 2.178 \\
\hline 1-hexyne & 2.091 & 1.941 & 1.802 & 1.000 & 2.506 \\
\hline 1-heptyne & 2.490 & 2.319 & 2.160 & 1.000 & 2.963 \\
\hline 2-butanone & 2.578 & 2.413 & 2.261 & 1.000 & 3.032 \\
\hline 2-pentanone & 2.932 & 2.750 & 2.584 & 1.000 & 3.432 \\
\hline 3-pentanone & 2.924 & 2.743 & 2.577 & 1.000 & 3.423 \\
\hline 1,4-dioxane & 2.781 & 2.607 & 2.446 & 1.000 & 3.263 \\
\hline methanol & 2.085 & 1.849 & 1.659 & 0.998 & 2.692 \\
\hline ethanol & 2.104 & 1.939 & 1.791 & 1.000 & 2.553 \\
\hline 1-propanol & 2.475 & 2.293 & 2.136 & 0.999 & 2.959 \\
\hline 2-propanol & 2.189 & 2.021 & 1.868 & 1.000 & 2.651 \\
\hline 2-methyl-1-propanol & 2.708 & 2.518 & 2.348 & 1.000 & 3.226 \\
\hline trifluoroethanol & 2.405 & 2.328 & 2.167 & 0.953 & 2.761 \\
\hline diethyl ether & 1.564 & 1.441 & 1.331 & 1.000 & 1.898 \\
\hline diisopropyl ether & 1.881 & 1.741 & 1.612 & 1.000 & 2.268 \\
\hline chloroform & 2.272 & 2.122 & 1.985 & 1.000 & 2.685 \\
\hline dichloromethane & 1.933 & 1.806 & 1.690 & 1.000 & 2.281 \\
\hline tetrachloromethane & 2.226 & 2.081 & 1.948 & 1.000 & 2.625 \\
\hline acetonitrile & 2.418 & 2.278 & 2.141 & 1.000 & 2.818 \\
\hline nitromethane & 2.650 & 2.497 & 2.356 & 1.000 & 3.073 \\
\hline 1-nitropropane & 3.258 & 3.065 & 2.901 & 0.999 & 3.769 \\
\hline triethylamine & 2.214 & 2.056 & 1.922 & 0.999 & 2.631 \\
\hline pyridine & 3.144 & 2.965 & 2.796 & 1.000 & 3.644 \\
\hline thiophene & 2.553 & 2.397 & 2.253 & 1.000 & 2.985 \\
\hline formaldehyde & 1.752 & 1.616 & 1.496 & 1.000 & 2.118 \\
\hline propionaldehyde & 2.210 & 1.929 & 1.798 & 0.965 & 2.783 \\
\hline butyraldehyde & & & 2.243 & & \\
\hline
\end{tabular}

accurate mathematical description of the $\log K_{\mathrm{L}}$ and $\log P$ values for organic solutes dissolved in $\left[\mathrm{MB}_{3} \mathrm{AM}\right]^{+}\left[\mathrm{Tf}_{2} \mathrm{~N}\right]^{-}$as evidenced by standard deviations of $\mathrm{SD}=0.083$ and $\mathrm{SD}=0.113 \mathrm{log}$ units. Standard deviations for the $\log P$ correlations are generally slightly larger because the values used in the regression analysis also include the 
Table 9. Selectivities $\left(S_{12}^{\infty}\right)$ and Capacities $\left(k_{1}^{\infty}\right)$ at Infinite Dilution for Different Separation Problems at $323.15 \mathrm{~K}$

\begin{tabular}{|c|c|c|c|c|c|c|}
\hline \multicolumn{2}{|r|}{ ILs } & \multicolumn{4}{|c|}{$S_{12}^{\infty} / k_{1}^{\infty}$} & \multirow[t]{2}{*}{ reference } \\
\hline Anion & Cation & Hexane/Benzene & Hexane/Methanol & Hexane/Thiophene & Cyclohexane/Thiophene & \\
\hline$\left[\mathrm{Tf}_{2} \mathrm{~N}\right]^{-}$ & methyltributylammonium & $7.5 / 1.11$ & $7.3 / 1.08$ & $7.8 / 1.17$ & $5.5 / 1.17$ & this work \\
\hline & octyltrimethylammonium & $8.5 / 1.37$ & $6.75 / 1.08$ & $9 / 1.45$ & $5.9 / 1.45$ & this work \\
\hline & decyltrimethylammonium & $5.9 / 1.41$ & $5.3 / 1.09$ & $6.46 / 1.54$ & $4.46 / 1.54$ & this work \\
\hline & tetraoctylammonium & $3.2 / 2.5$ & $2.0 / 1.08$ & $3.25 / 2.5$ & $2.35 / 2.5$ & this work \\
\hline & 1,3-dimethoxyimidazolium & $21.3 / 0.47$ & $42.05 / 0.94$ & $24.8 / 0.94$ & $12.6 / 0.94$ & 18 \\
\hline & 1-(methylethylether)-3-methylimidazolium & $15.5 / 0.85$ & $17.4 / 0.93$ & $18.1 / 1.0$ & $10.9 / 1.0$ & 18 \\
\hline & 1-ethanol-3-methylimidazolium & $20.6 / 0.47$ & $49.1 / 1.12$ & $24.7 / 0.56$ & $14.2 / 0.56$ & 18 \\
\hline & 1-ethyl-3-methylimidazolium & $37.5 / 1.43$ & $19.5 / 1.20$ & & & 32 \\
\hline & 1-(hexylmethylether)-3-methylimidazolium & $9.1 / 1.23$ & $6.8 / 0.91$ & $10.0 / 1.35$ & $6.4 / 1.35$ & 33 \\
\hline & 1,3-bis(hexylmethylether)imidazolium & $4.9 / 1.67$ & $3.2 / 1.06$ & $5.3 / 1.75$ & $3.7 / 1.75$ & 33 \\
\hline & 1-butyl-3-methylimidazolium & $16.7 / 1.11$ & & & & 34 \\
\hline & 1-hexyl-3-methylimidazolium & $9.5 / 1.29$ & $6.1 / 0.82$ & & & 35 \\
\hline & trimethylhexylammonium & $9.9 / 1.01$ & $8.5 / 0.86$ & $10.7 / 1.09$ & $7.2 / 1.09$ & 15 \\
\hline & 4-methyl- $N$-butylpyridinium & $18.8 / 1.43$ & $21.2 / 0.83$ & $10.6 / 1.56$ & $6.1 / 1.56$ & 36 \\
\hline & triethylsulfonium & $21.6 / 0.91$ & $17.8 / 0.77$ & $25.5 / 1.05$ & $14.3 / 1.05$ & 37 \\
\hline & trihexyl(tetradecyl) phosphonium & $2.7 / 2.56$ & $1.1 / 1.02$ & $2.6 / 2.5$ & $1.95 / 2.5$ & 4 \\
\hline
\end{tabular}

Table 10. Equation Coefficients for the Abraham Model $\log K_{\mathrm{L}}$ and $\log P$ Correlations for $\left[\mathrm{MB}_{3} \mathrm{AM}^{+}\left[\mathrm{Tf}_{2} \mathrm{~N}\right]^{-}\right.$, $\left[\mathrm{OM}_{3} \mathrm{AM}\right]^{+}\left[\mathrm{Tf}_{2} \mathbf{N}\right]^{-},\left[\mathrm{DM}_{3} \mathrm{AM}\right]^{+}\left[\mathrm{Tf}_{2} \mathbf{N}\right]^{-}$, and $\left[\mathrm{O}_{4} \mathrm{AM}\right]^{+}\left[\mathrm{Tf}_{2} \mathbf{N}\right]^{-}$

\begin{tabular}{|c|c|c|c|c|c|c|c|c|c|c|c|}
\hline IL & property & $c$ & $e$ & $s$ & $a$ & $b$ & $v / l$ & $N$ & SD & $R^{2}$ & $F$ \\
\hline$\left[\mathrm{MB}_{3} \mathrm{Am}\right]^{+}\left[\mathrm{Tf}_{2} \mathrm{~N}\right]^{-}$ & $\log K_{\mathrm{L}}$ & $\begin{array}{r}-0.506 \\
(0.079)\end{array}$ & $\begin{array}{r}-0.169 \\
(0.083)\end{array}$ & $\begin{array}{c}2.103 \\
(0.080)\end{array}$ & $\begin{array}{c}2.298 \\
(0.115)\end{array}$ & $\begin{array}{c}0.412 \\
(0.092)\end{array}$ & $\begin{array}{c}0.777 \\
(0.022)\end{array}$ & 44 & 0.083 & 0.983 & 452.1 \\
\hline$\left[\mathrm{MB}_{3} \mathrm{Am}\right]^{+}\left[\mathrm{Tf}_{2} \mathrm{~N}\right]^{-}$ & $\log P$ & $\begin{array}{l}-0.233 \\
(0.123)\end{array}$ & 0.000 & $\begin{array}{c}0.404 \\
(0.101)\end{array}$ & $\begin{array}{r}-1.313 \\
(0.154)\end{array}$ & $\begin{array}{l}-4.542 \\
(0.113)\end{array}$ & $\begin{array}{c}3.687 \\
(0.104)\end{array}$ & 44 & 0.113 & 0.996 & 2551 \\
\hline$\left[\mathrm{OM}_{3} \mathrm{AM}\right]^{+}\left[\mathrm{Tf}_{2} \mathrm{~N}\right]^{-}$ & $\log K_{\mathrm{L}}$ & $\begin{array}{r}-0.426 \\
(0.082)\end{array}$ & $\begin{array}{r}-0.338 \\
(0.089)\end{array}$ & $\begin{array}{c}2.242 \\
(0.082)\end{array}$ & $\begin{array}{c}2.195 \\
(0.123)\end{array}$ & $\begin{array}{c}0.684 \\
(0.021)\end{array}$ & $\begin{array}{c}0.779 \\
(0.022)\end{array}$ & 44 & 0.092 & 0.981 & 396.5 \\
\hline$\left[\mathrm{OM}_{3} \mathrm{AM}\right]^{+}\left[\mathrm{Tf}_{2} \mathrm{~N}\right]^{-}$ & $\log P$ & $\begin{array}{r}-0.165 \\
(0.129)\end{array}$ & $\begin{array}{r}-0.181 \\
(0.118)\end{array}$ & $\begin{array}{c}0.569 \\
(0.118)\end{array}$ & $\begin{array}{r}-1.419 \\
(0.164)\end{array}$ & $\begin{array}{r}-4.677 \\
(0.115)\end{array}$ & $\begin{array}{c}3.711 \\
(0.103)\end{array}$ & 44 & 0.123 & 0.996 & 1976 \\
\hline$\left[\mathrm{DM}_{3} \mathrm{AM}\right]^{+}\left[\mathrm{Tf}_{2} \mathrm{~N}\right]^{-}$ & $\log K_{\mathrm{L}}$ & $\begin{array}{r}-0.363 \\
(0.089)\end{array}$ & $\begin{array}{r}-0.339 \\
(0.098)\end{array}$ & $\begin{array}{c}1.986 \\
(0.090)\end{array}$ & $\begin{array}{c}2.144 \\
(0.135)\end{array}$ & $\begin{array}{c}0.422 \\
(0.094)\end{array}$ & $\begin{array}{c}0.809 \\
(0.024)\end{array}$ & 46 & 0.102 & 0.974 & 303.2 \\
\hline$\left[\mathrm{DM}_{3} \mathrm{AM}\right]^{+}\left[\mathrm{Tf}_{2} \mathrm{~N}\right]^{-}$ & $\log P$ & $\begin{array}{r}-0.128 \\
(0.135)\end{array}$ & $\begin{array}{r}-0.131 \\
(0.125)\end{array}$ & $\begin{array}{c}0.329 \\
(0.126)\end{array}$ & $\begin{array}{r}-1.458 \\
(0.175)\end{array}$ & $\begin{array}{r}-4.550 \\
(0.120)\end{array}$ & $\begin{array}{c}3.816 \\
(0.108)\end{array}$ & 46 & 0.132 & 0.996 & 1832 \\
\hline$\left[\mathrm{O}_{4} \mathrm{AM}\right]^{+}\left[\mathrm{Tf}_{2} \mathrm{~N}\right]^{-}$ & $\log K_{\mathrm{L}}$ & 0.000 & $\begin{array}{r}-0.287 \\
(0.134)\end{array}$ & $\begin{array}{c}1.478 \\
(0.110)\end{array}$ & $\begin{array}{c}1.845 \\
(0.158)\end{array}$ & $\begin{array}{c}0.189 \\
(0.121)\end{array}$ & $\begin{array}{c}0.816 \\
(0.013)\end{array}$ & 42 & 0.124 & 0.998 & 3749 \\
\hline$\left[\mathrm{O}_{4} \mathrm{AM}\right]^{+}\left[\mathrm{Tf}_{2} \mathrm{~N}\right]^{-}$ & $\log P$ & $\begin{array}{c}0.226 \\
(0.198)\end{array}$ & 0.000 & $\begin{array}{c}-0.212 \\
(0.144)\end{array}$ & $\begin{array}{r}-1.756 \\
(0.240)\end{array}$ & $\begin{array}{l}-4.739 \\
(0.139)\end{array}$ & $\begin{array}{c}3.825 \\
(0.174)\end{array}$ & 42 & 0.164 & 0.993 & 1332 \\
\hline
\end{tabular}

experimental uncertainty in the $\log K_{\mathrm{w}}$ values used to convert the measured $\log K_{\mathrm{L}}$ data to $\log P$.

As noted above, each of the calculated equation coefficients corresponds to the sum of the respective cation-specific and anionspecific contributions. In establishing the computational methodology, the equation coefficients for the $\left[\mathrm{Tf}_{2} \mathrm{~N}\right]^{-}$anion were set equal to zero to provide a reference point from which all equation coefficients would be calculated. ${ }^{28-30}$ A reference point is needed because the cation- and anion-specific equation coefficients are generated as a paired set. The $\log K_{\mathrm{L}}$ and $\log P$ equation coefficients for $\left[\mathrm{MB}_{3} \mathrm{AM}\right]^{+}\left[\mathrm{Tf}_{2} \mathrm{~N}\right]^{-}$thus correspond to the ionspecific coefficients for the $\left[\mathrm{MB}_{3} \mathrm{AM}\right]^{+}$cation.

Tabulated in Table 3 are 44 experimental $\log K_{\mathrm{L}}$ and $\log P$ values for solutes dissolved in $\left[\mathrm{OM}_{3} \mathrm{AM}\right]^{+}\left[\mathrm{Tf}_{2} \mathrm{~N}\right]^{-}$. The calculated Abraham model equation coefficients for transfer of organic solutes to the
IL both from the gas phase and from water are given in Table 10. The statistics of both correlations are quite good as evidenced by squared correlation coefficients near unity and small standard deviations of 0.092 and $0.123 \log$ units. The $\log K_{\mathrm{L}}$ and $\log P$ equation coefficients correspond to the ion-specific coefficients for the $\left[\mathrm{OM}_{3} \mathrm{AM}\right]^{+}$cation because the anion-specific values for the $\left[\mathrm{Tf}_{2} \mathrm{~N}\right]^{-}$anion were defined to be zero, as discussed above.

The experimental $\log K_{\mathrm{L}}$ and $\log P$ data in Tables 5 and 7 for $\left[\mathrm{DM}_{3} \mathrm{AM}\right]^{+}\left[\mathrm{Tf}_{2} \mathrm{~N}\right]^{-}$and $\left[\mathrm{O}_{4} \mathrm{AM}\right]^{+}\left[\mathrm{Tf}_{2} \mathrm{~N}\right]^{-}$were analyzed in a similar fashion to give the equation coefficients listed in Table 10. Preliminary regression analysis showed the $c$ term in the $\log K_{\mathrm{L}}$ correlation for $\left[\mathrm{O}_{4} \mathrm{AM}\right]^{+}\left[\mathrm{Tf}_{2} \mathrm{~N}\right]^{-}$and the $e \cdot E$ term in the $\log P$ correlation for $\left[\mathrm{O}_{4} \mathrm{AM}\right]^{+}\left[\mathrm{Tf}_{2} \mathrm{~N}\right]^{-}$to be negligible; both terms were thus removed from the final set of equations. The derived Abraham model correlations provide a good mathematical description of the 
Table 11. Solute Descriptors of Organic Compounds Considered in this Study

\begin{tabular}{|c|c|c|c|c|c|c|}
\hline solute & $E$ & $S$ & $A$ & $B$ & $L$ & $V$ \\
\hline hexane & 0.000 & 0.000 & 0.000 & 0.000 & 2.668 & 0.9540 \\
\hline 3-methylpentane & 0.000 & 0.000 & 0.000 & 0.000 & 2.581 & 0.9540 \\
\hline heptane & 0.000 & 0.000 & 0.000 & 0.000 & 3.173 & 1.0949 \\
\hline 2,2,4-trimethylpentane & 0.000 & 0.000 & 0.000 & 0.000 & 3.106 & 1.2358 \\
\hline octane & 0.000 & 0.000 & 0.000 & 0.000 & 3.677 & 1.2358 \\
\hline nonane & 0.000 & 0.000 & 0.000 & 0.000 & 4.182 & 1.3767 \\
\hline decane & 0.000 & 0.000 & 0.000 & 0.000 & 4.686 & 1.5176 \\
\hline undecane & 0.000 & 0.000 & 0.000 & 0.000 & 5.191 & 1.6590 \\
\hline dodecane & 0.000 & 0.000 & 0.000 & 0.000 & 5.696 & 1.7994 \\
\hline tridecane & 0.000 & 0.000 & 0.000 & 0.000 & 6.200 & 1.9400 \\
\hline tetradecane & 0.000 & 0.000 & 0.000 & 0.000 & 6.705 & 2.0810 \\
\hline methylcyclopentane & 0.225 & 0.100 & 0.000 & 0.000 & 2.907 & 0.8454 \\
\hline cyclohexane & 0.310 & 0.100 & 0.000 & 0.000 & 2.964 & 0.8454 \\
\hline methylcyclohexane & 0.244 & 0.060 & 0.000 & 0.000 & 3.319 & 0.9863 \\
\hline cycloheptane & 0.350 & 0.100 & 0.000 & 0.000 & 3.704 & 0.9863 \\
\hline benzene & 0.610 & 0.520 & 0.000 & 0.140 & 2.786 & 0.7164 \\
\hline toluene & 0.601 & 0.520 & 0.000 & 0.140 & 3.325 & 0.8573 \\
\hline ethylbenzene & 0.613 & 0.510 & 0.000 & 0.150 & 3.778 & 0.9982 \\
\hline$m$-xylene & 0.623 & 0.520 & 0.000 & 0.160 & 3.839 & 0.9982 \\
\hline$p$-xylene & 0.613 & 0.520 & 0.000 & 0.160 & 3.839 & 0.9982 \\
\hline$o$-xylene & 0.663 & 0.560 & 0.000 & 0.160 & 3.939 & 0.9982 \\
\hline 1-hexene & 0.080 & 0.080 & 0.000 & 0.070 & 2.572 & 0.9110 \\
\hline 1-hexyne & 0.166 & 0.220 & 0.100 & 0.120 & 2.510 & 0.8680 \\
\hline 1-heptyne & 0.160 & 0.230 & 0.090 & 0.100 & 3.000 & 1.0089 \\
\hline 2-butanone & 0.166 & 0.700 & 0.000 & 0.510 & 2.287 & 0.6879 \\
\hline 2-pentanone & 0.143 & 0.680 & 0.000 & 0.510 & 2.755 & 0.8288 \\
\hline 3-pentanone & 0.154 & 0.660 & 0.000 & 0.510 & 2.811 & 0.8288 \\
\hline 1,4 dioxane & 0.329 & 0.750 & 0.000 & 0.640 & 2.892 & 0.6810 \\
\hline methanol & 0.278 & 0.440 & 0.430 & 0.470 & 0.970 & 0.3082 \\
\hline ethanol & 0.246 & 0.420 & 0.370 & 0.480 & 1.485 & 0.4491 \\
\hline 1-propanol & 0.236 & 0.420 & 0.370 & 0.480 & 2.031 & 0.5900 \\
\hline 2-propanol & 0.212 & 0.360 & 0.330 & 0.560 & 1.764 & 0.5900 \\
\hline 2-methyl-1-propanol & 0.217 & 0.390 & 0.370 & 0.480 & 2.413 & 0.7309 \\
\hline 1-butanol & 0.224 & 0.420 & 0.370 & 0.480 & 2.601 & 0.7309 \\
\hline trifluoroethanol & 0.015 & 0.600 & 0.570 & 0.250 & 1.224 & 0.5022 \\
\hline diethyl ether & 0.041 & 0.250 & 0.000 & 0.450 & 2.015 & 0.7309 \\
\hline diisopropyl ether & -0.063 & 0.170 & 0.000 & 0.570 & 2.501 & 1.0127 \\
\hline chloroform & 0.425 & 0.490 & 0.150 & 0.020 & 2.480 & 0.6167 \\
\hline dichloromethane & 0.390 & 0.570 & 0.100 & 0.050 & 2.019 & 0.4943 \\
\hline tetrachloromethane & 0.460 & 0.380 & 0.000 & 0.000 & 2.823 & 0.7391 \\
\hline acetonitrile & 0.237 & 0.900 & 0.070 & 0.320 & 1.739 & 0.4042 \\
\hline nitromethane & 0.313 & 0.950 & 0.060 & 0.310 & 1.892 & 0.4237 \\
\hline 1-nitropropane & 0.242 & 0.950 & 0.000 & 0.310 & 2.894 & 0.7055 \\
\hline triethylamine & 0.101 & 0.150 & 0.000 & 0.790 & 3.040 & 1.0538 \\
\hline pyridine & 0.631 & 0.840 & 0.000 & 0.520 & 3.022 & 0.6753 \\
\hline thiophene & 0.687 & 0.570 & 0.000 & 0.150 & 2.819 & 0.6411 \\
\hline propionaldehyde & 0.196 & 0.650 & 0.000 & 0.450 & 1.815 & 0.5470 \\
\hline butyraldehyde & 0.187 & 0.650 & 0.000 & 0.450 & 2.270 & 0.6879 \\
\hline
\end{tabular}

experimental gas-to-anhydrous IL partition coefficient data and water-to-anhydrous IL partition coefficient.

The Abraham model correlations that have been derived for $\left[\mathrm{MB}_{3} \mathrm{AM}\right]^{+}\left[\mathrm{Tf}_{2} \mathrm{~N}\right]^{-},\left[\mathrm{OM}_{3} \mathrm{AM}\right]^{+}\left[\mathrm{Tf}_{2} \mathrm{~N}\right]^{-},\left[\mathrm{DM}_{3} \mathrm{AM}\right]^{+}\left[\mathrm{Tf}_{2} \mathrm{~N}\right]^{-}$, and $\left[\mathrm{O}_{4} \mathrm{AM}\right]^{+}\left[\mathrm{Tf}_{2} \mathrm{~N}\right]^{-}$would be expected to provide very reasonable predictions for the $\log K_{\mathrm{L}}$ values for additional solutes having descriptor values that fall within the range of chemical space defined by $E=-0.063$ to $0.687, S=0.000$ to $0.950, A=0.000$ to 0.570 , $B=0.000$ to $0.790, L=0.970$ to 6.705 , and $V=0.308$ to 2.081 . The cation- and anion-specific equation coefficients can be combined to yield predictive equations for many different ILs. The present study increases our calculated cation-specific equation coefficients by 4 . We have now reported equation coefficients for 24 unique cations, counting the 4 tetraalkylammonium cations introduced here, and for 14 different anions. The 24 sets of cation-specific equation coefficients and 14 sets of anion-specific equation coefficients can be combined to yield predictive equations for 336 different ILs.

\section{AUTHOR INFORMATION}

\section{Corresponding Author}

*E-mail: acree@unt.edu.

\section{REFERENCES}

(1) Saravanamurugan, S.; Due-Hansen, J.; Kegnoes, S.; Gretarsdottir, T.; Riisager, A.; Fehrmann, R. Ionic liquid gas absorption of $\mathrm{NO}_{x}, \mathrm{CO}_{\mathrm{x}}$ and $\mathrm{SO}_{\mathrm{x}}$. ACS Symp. Ser., Div. Fuel Chem. 2010, 55 (2), 6.

(2) Revelli, A.-L.; Mutelet, F.; Jaubert, J.-N. Prediction of partition coefficients of organic compounds in ionic liquids: Use of a linear solvation energy relationship with parameters calculated through a group contribution method. Ind. Eng. Chem. Res. 2010, 49, 3883-3892.

(3) Meindersma, G. W.; Galan Sanchez, L. M.; Hansmeier, A. R.; De Haan, A. B. Application of task-specific ionic liquids for intensified separations. Monatsh. Chem. 2007, 138, 1125-1136.

(4) Revelli, A.-L.; Sprunger, L. M.; Gibbs, J.; Acree, W. E.; Baker, G. A.; Mutelet, F. Activity coefficients at infinite dilution of organic compounds in trihexyl(tetradecyl)phosphonium bis(trifluoromethylsulfonyl)imide using inverse gas chromatography. J. Chem. Eng. Data 2009, 54, 977-985.

(5) Revelli, A.-L.; Mutelet, F.; Turmine, M.; Solimando, R.; Jaubert, J.-N. Activity coefficients at infinite dilution of organic compounds in 1-butyl-3-methylimidazolium tetrafluoroborate using inverse gas chromatography. J. Chem. Eng. Data 2008, 54, 90-101.

(6) Mutelet, F.; Butet, V.; Jaubert, J.-N. Application of inverse gas chromatography and regular solution theory for characterization of ionic liquids. Ind. Eng. Chem. Res. 2005, 44, 4120-4127.

(7) Mutelet, F.; Jaubert, J.-N. Accurate measurements of thermodynamic properties of solutes in ionic liquids using inverse gas chromatography. J. Chromatogr., A 2006, 1102, 256-267.

(8) Mutelet, F.; Jaubert, J.-N.; Rogalski, M.; Boukherissa, M.; Dicko, A. Thermodynamic properties of mixtures containing ionic liquids: activity coefficients at infinite dilution of organic compounds in 1-propyl boronic acid-3-alkylimidazolium bromide and 1-propenyl-3-alkylimidazolium bromide using inverse gas chromatography. J. Chem. Eng. Data 2006, 51, 1274-1279.

(9) Mutelet, F.; Jaubert, J.-N. Measurement of activity coefficients at infinite dilution in 1-hexadecyl-3-methylimidazolium tetrafluoroborate ionic liquid. J. Chem. Thermodyn. 2007, 39, 1144-1150.

(10) Mutelet, F.; Jaubert, J.-N.; Rogalski, M.; Harmand, J.; Sindt, M.; Mieloszynski, J.-L. Activity coefficients at infinite dilution of organic compounds in 1-methacryloyloxyalkyl-3-methylimidazolium bromide using inverse gas chromatography. J. Phys. Chem. B 2008, 112, 3773-3785.

(11) Acree, W. E., Jr.; Abraham, M. H. The analysis of solvation in ionic liquids and organic solvents using the Abraham linear free energy relationship. J. Chem. Technol. Biotechnol., 2006, 81, 1441-1446. [Erratum: J. Chem. Technol. Biotechnol. 2006, 81, 1722].

(12) Abraham, M. H.; Acree, W. E., Jr. Comparative analysis of solvation and selectivity in room temperature ionic liquids using the Abraham linear free energy relationship. Green Chem. 2006, 8, 906-915. 
(13) Mintz, C.; Acree, W. E., Jr. Partition coefficient correlations for transfer of solutes from gas phase and water to room temperature ionic liquids. Phys. Chem. Liq. 2007, 45, 241-249.

(14) Sprunger, L. M.; Acree, W. E., Jr.; Abraham, M. H. Linear free energy relationship correlations for the solubilising characterisation of room temperature ionic liquids containing 1-hexyloxymethyl-3-methylimidazolium and 1,3-dihexyloxymethyl-imidazolium cations. Phys. Chem. Liq. 2010, 48, 394-402.

(15) Mutelet, F.; Revelli, A.-L.; Jaubert, J.-N.; Sprunger, L. M.; Acree, W. E., Jr.; Baker, G. A. Partition coefficients of organic compounds in new imidazolium and tetralkylammonium based ionic liquids using inverse gas chromatography. J. Chem. Eng. Data 2010, 55, 234-242.

(16) Sprunger, L. M.; Gibbs, J.; Baltazar, Q. Q.; Acree, W. E., Jr.; Abraham, M. H.; Anderson, J. L. Characterisation of room temperature ionic liquid chromatographic stationary phases by combining experimental retention factor and partition coefficient data into a single model. Phys. Chem. Liq. 2009, 47, 74-83.

(17) Sprunger, L. M.; Acree, W. E., Jr.; Abraham, M. H. Linear free energy relationship (LFER) correlations for the solubilising characterisation of room temperature ionic liquids containing triethylsulphonium and 1-butyl-1-methylpyrrolidinium cations. Phys. Chem. Liq. 2010, 48, 385-393.

(18) Revelli, A.-L.; Mutelet, F.; Jaubert, J.-N.; Garcia-Martinez, M.; Sprunger, L. M.; Acree, W. E., Jr.; Baker, G. A. Study of ether-, alcohol-, or cyano-functionalized ionic liquids using inverse gas chromatography. J. Chem. Eng. Data 2010, 55, 2434-2443.

(19) Grubbs, L. M.; Ye, S.; Saifullah, M.; Acree, W. E., Jr.; Twu, P.; Anderson, J. L.; Baker, G. A.; Abraham, M. H. correlation of the solubilizing abilities of hexyl(trimethyl)-ammonium bis((trifluoromethyl)sulfonyl) imide, 1-propyl-1-methylpiperidinium bis((trifluoromethyl)-sulfonyl)imide and 1-butyl-1-methyl-pyrrolidinium thiocyanate. J. Solution Chem. 2011, in press.

(20) Grubbs, L. M.; Saifullah, M.; De La Rosa, N. E.; Acree, W. E., Jr.; Abraham, M. H.; Zhao, Q.; Anderson, J. L. Cation-specific and anionspecific Abraham model correlations for solute transfer into ionic liquids. Global J. Phys. Chem. 2010, 1, 1-19.

(21) Proctor, A.; Sprunger, L. M.; Acree, W. E., Jr.; Abraham, M. H. LFER correlations for the solubilising characterisation of room temperature ionic liquids containing trifluoro-methanesulfonate and trifluoroacetate anions. Phys. Chem. Liq. 2008, 46, 631-642.

(22) Moise, J. C.; Mutelet, F.; Jaubert, J.-N.; Grubbs, L. M.; Acree, W. E., Jr.; Baker, G. A. Activity coefficients at infinite dilution of organic compounds in four new imidazolium-based ionic liquids. J. Chem. Eng. Data 2011, 56, 3106-3114.

(23) Abraham, M. H.; Zissimos, A. M.; Huddleston, J. G.; Willauer, H. D.; Rogers, R. D.; Acree, W. E., Jr. Some novel liquid partitioning systems: water-ionic liquids and aqueous biphasic systems. Ind. Eng. Chem. Res. 2003, 42, 413-418.

(24) Sprunger, L.; Clark, M.; Acree, W. E., Jr.; Abraham, M. H. Characterization of room-temperature ionic liquids by the Abraham model with cation-specific and anion-specific equation coefficients. J. Chem. Inf. Model. 2007, 47, 1123-1129.

(25) Sprunger, L. M.; Proctor, A.; Acree, W. E., Jr.; Abraham, M. H. LFER correlations for room temperature ionic liquids: Separation of equation coefficients into individual cation-specific and anion-specific contributions. Fluid Phase Equilib. 2008, 265, 104-111.

(26) Sprunger, L. M.; Gibbs, J.; Proctor, A.; Acree, W. E., Jr.; Abraham, M. H.; Meng, Y.; Yao, C.; Anderson, J. L. Linear free energy relationship correlations for room temperature ionic liquids: revised cation-specific and anion-specific equation coefficients for predictive applications covering a much larger area of chemical space. Ind. Eng. Chem. Res. 2009, 48, 4145-4154.

(27) Kilaru, P.; Baker, G. A.; Scovazzo, P. Density and Surface Tension Measurements of Imidazolium-, Quaternary Phosphonium-, and Ammonium-Based Room-Temperature Ionic Liquids: Data and Correlations. J. Chem. Eng. Data 2007, 52, 2306-2314.

(28) Burrell, A. K.; Del Sesto, R. E.; Baker, S. N.; McCleskey, T. M.; Baker, G. A. The Large Scale Synthesis of Pure Imidazolium and Pyrrolidinium Ionic Liquids. Green Chem. 2007, 9, 449-454.
(29) Luo, H. M.; Baker, G. A.; Dai, S. Isothermogravimetric determination of the enthalpies. J. Phys. Chem. B 2008, 112, 10077-10081.

(30) Jin, H.; O'Hare, B.; Dong, J.; Arzhantsev, S.; Baker, G. A.; Wishart, J. F.; Benesi, A. J.; Maroncelli, M. Physical properties of ionic liquids consisting of the 1-butyl-3-methylimidazolium cation with various anions and the bis(trifluoromethylsulfonyl)imide anion with various cations. J. Phys. Chem. B 2008, 112, 81-92.

(31) Cruickshank, A. J. B.; Windsor, M. L.; Young, C. L. The use of gas-liquid chromatography to determine activity coefficients and second virial coefficients of mixtures. Proc. R. Soc. London 1966, A295, 259-270.

(32) Deenadayalu, N.; Letcher, T. M.; Reddy, P. Determination of activity coefficients at infinite dilution of polar and nonpolar solutes in the ionic liquid 1-ethyl-3-methylimidazolium bis(trifluoromethylsulfonyl) imidate using gas-liquid chromatography at the temperature 303.15 or 318.15 K. J. Chem. Eng. Data 2005, 50, 105-108.

(33) Domanska, U.; Marciniak, A. Activity coefficients at infinite dilution measurements for organic solutes and water in the 1-hexyloxymethyl-3-methyl-imidazolium and 1,3-dihexyloxy-methylimidazolium bis(trifluoromethylsulfonyl)imide ionic liquids. The cation influence. Fluid Phase Equilib. 2009, 286, 154-161.

(34) Krummen, M.; Wasserscheid, P.; Gmehling, J. Measurement of activity coefficients at infinite dilution in ionic liquids using the dilutor technique. J. Chem. Eng. Data 2002, 7, 1411-1417.

(35) Letcher, T. M.; Marciniak, A.; Marciniak, M.; Domanska, U. Activity coefficients at infinite dilution measurements for organic solutes in the ionic liquid 1-hexyl-3-methyl-imidazolium bis(trifluoromethylsulfonyl)-imide using g.l.c. at $T=(298.15,313.15$, and 333.15) K. J. Chem. Thermodyn. 2005, 37, 1327-1331.

(36) Domanska, U.; Marciniak, A. Activity coefficients at infinite dilution measurements for organic solutes and water in the ionic liquid 4-methyl-N-butyl-pyridinium bis(trifluoromethyl-sulfonyl)imide. J. Chem. Thermodyn. 2009, 41, 1350-1355.

(37) Domanska, U.; Marciniak, A. Activity coefficients at infinite dilution measurements for organic solutes and water in the ionic liquid triethylsulphonium bis(trifluoromethylsulfonyl)-imide. J. Chem. Thermodyn. 2009, 41, 754-758.

(38) Letcher, T. M.; Marciniak, A.; Marciniak, M.; Domanska, U. Determination of activity coefficients at infinite dilution of solutes in the ionic liquid 1-butyl-3-methylimidazolium octyl sulfate using gas-liquid chromatography at a temperature of $298.15 \mathrm{~K}, 313.15 \mathrm{~K}$, or $328.15 \mathrm{~K}$. J. Chem. Eng. Data 2005, 50, 1294-1298. 